\title{
Comparison of Two Retention Appliances with Respect to Clinical Effectiveness
}

\author{
Yeşim Kaya' (D), Murat Tunca' (D), Sıddık Keskin²
}

'Department of Orthodontics, Yüzüncü Yıl University School of Dentistry, Van, Turkey 2Department of Biostatistics, Yüzüncü Yıl University School of Dentistry, Van, Turkey

\section{ABSTRACT}

Objective: The aim of this study was to compare the clinical effectiveness of Essix and Hawley retainers during the retention period. Methods: A total of 30 subjects whose fixed orthodontic treatment results were evaluated according to the American Board of Orthodontics Phase III Objective Grading system were included in this study. After the removal of orthodontic attachments, the study participants were equally divided into two retention protocols: upper-lower Essix and upper-lower Hawley. The subjects were instructed to wear their retainers full time for 6 months, except during meals, and during nights only for 6 months. The clinical effectiveness of the retainers was evaluated according to the overjet, overbite, maxillary, and mandibular intercanine widths, intermolar widths, arch lengths, irregularity indexes, and lateral cephalometric measurements. All dental model and lateral cephalometric measurements were performed by the same investigator during three periods: pre-treatment, post-treatment, and post-retention.

Results: The overjet, overbite, maxillary, and mandibular intercanine widths; intermolar widths; and arch lengths and lateral cephalometric measurements were not statistically significantly different between the groups and identified time periods. Although the maxillary and mandibular irregularity indexes increased from the post-treatment to post-retention periods, the difference was not statistically significant. Pre-treatment, post-treatment, and post-retention lateral cephalometric measurements were not statistically significantly different between and within the groups.

Conclusion: According to the results of a repeated-measures analysis of variance with two factors, and although an increase was found in the maxillary and mandibular irregularity indexes, the clinical effectiveness of Essix and Hawley retainers was found to be similar during the retention period.

Keywords: Orthodontic treatment, retention, Essix, Hawley, relapse

\section{INTRODUCTION}

A long-term stability of the treatment results obtained at the end of active orthodontic treatment is one of the success indicators of orthodontic treatment (1). Stability can only be achieved when the forces derived from the gingival and periodontal tissues, orofacial soft tissues, occlusion, and post-treatment facial growth and development are balanced (2). At this point, it is very important to determine the necessities of the retention phase and the factors that cause a relapse in terms of stability (3).

Relapse is defined as the return of dental and skeletal results, obtained aesthetically and functionally, to the pre-treatment status at the end of the active orthodontic treatment (4). The major requirement to prevent a relapse is time to reorganize the gingival and periodontal tissues and stabilize the altered morphological structure and function and growth-development dependent changes $(4,5)$. For this reason, retainers are used to prevent a relapse after active orthodontic treatment (6). 
Retainers used in the retention phase are divided into two groups; that is, removable and fixed $(4,7)$. While the removable retainers are classified as Essix, Hawley, and Positioner, the fixed retainers are classified as polyethylene and fiber-reinforced resin composites; currently, the most preferred multistranded stainless steel wires are those recommended by Zachrisson in 1977 $(4,8-10)$.

Although there is as yet no consensus as to which retainer is the most effective or how long it needs to be worn, currently, the Essix and Hawley retainers are frequently used in orthodontic practice $(6,11)$. It has been observed that the comparative studies of Essix and Hawley retainers have evaluated the periodontal health and compliance (12), cost-effectiveness (13), the number of occlusal contacts (14), survival time (15), and clinical effectiveness $(16,17)$. Some of the studies that evaluated the clinical effectiveness, such as the overjet, overbite, intercanine, and intermolar widths, arch length, and irregularity index, have indicated no significant difference between the two retainers $(16,18,19)$. It has also been stated that there is not enough evidence to declare which retainer is more effective $(5,6)$. In addition, Sheridan et al. (20) reported the retaining component of Hawley retainers is insufficient for anterior teeth due to an inadequate griping with a point contact on the vestibular arch on the labial surface and a mass of acrylic approximating the cervix.

The aim of the present study was to compare the clinical effectiveness of Essix and Hawley retainers, which are frequently used in orthodontic practice, during 1 year of the retention period. The null hypothesis was that the clinical effectiveness of Essix and Hawley retainers does not change with the appliance used.

\section{METHODS}

A total of 30 patients who underwent fixed orthodontic treatment with the straight-wire technique using 0.018-inch slot Roth brackets were enrolled in this study. The inclusion criteria were a Class I skeletal pattern, no previous orthodontic treatment, treatment with fixed orthodontic appliances, achievement of optimum occlusion, and treatment that was compatible with the use of a retainer and long-term follow-up, as well as good oral hygiene. The exclusion criteria were the necessity of using a bonded retainer and placement of a contemporary tooth in the retainer due to congenital tooth deficiency, cleft lip and plate, and orthognathic surgery.

This study protocol was approved by Yüzüncü Yıl University School of Medicine, Research Ethics Committee (B.30.2.YYU.0.01.00.00/125). Before debonding, the treatment outcomes were evaluated according to the objective grading system of the American Board of Orthodontics Phase III clinical examination. Informed consent was received from the patients, or their parents, who were to be included in this study after detailed information was given about the study.

After mechanical removal of the fixed orthodontic appliances with a debonding plier (Dentaurum, Pforzheim, Germany), residual adhesive on the tooth surfaces was cleaned with a 12-blad- ed tungsten carbide bur (Axis Dental, Irving, Tex) at low speed under water-cooling. Then, tooth surfaces were polished with fluoride-free pumice (Imıpomza, Imıcryl, Konya, Turkey), and alginate impressions were poured to obtain dental models of the upper and lower jaws.

The study participants were divided into two groups, depending on the type of retainers. Fifteen patients (8 extraction and 7 non-extraction) had an upper-lower Essix retainer (Dentsply Raintree Essix, New Orleans, Louisiana, USA), and 15 (7 extraction and 8 non-extraction) received an upper-lower Hawley retainer. Each group consisted of both extraction and non-extraction cases. The retainer type for each patient was randomly allocated by the technician.

Essix retainers were thermoformed from 0.040 -inch sheets according to the manufacturer's instructions. The retainer that covered up all occlusal surfaces, including the most distal tooth, was trimmed to provide 1-2 $\mathrm{mm}$ buccal and 3-4 $\mathrm{mm}$ lingual extensions that pass away from the edge of the gingiva (Figure 1a). A Hawley retainer was constructed from Adams clasps on the first molars, canine-canine teeth labial bows, and acrylic base plates. Adams clasps and labial bows were made from $0.7 \mathrm{~mm}$ stainless steel wire (Figure 1b). The patients were instructed to wear their retainers full-time for 6 months except during meals, and then 6 months at night only.

The retention characteristics of Essix and Hawley retainers were compared from lateral cephalometric film, and dental models

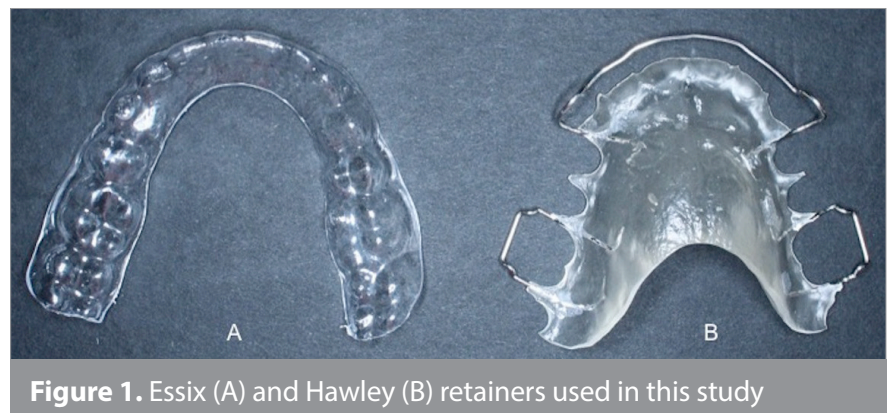

Table 1. Lateral cephalometric measurements

\section{Angular and Linear Measurements}

1-NA (mm) Distance between the most labial point of maxillary incisor and the NA line

1-NA $\left(^{\circ} \quad\right.$ Angle formed between the long axis of maxillary incisor and the NA line

1-SN $\left(^{\circ} \quad\right.$ Angle formed by the extension of the long axis of maxillary incisor to the SN plane

1-NB $(\mathrm{mm}) \quad$ Distance between the most labial point of mandibular incisor and the NB line

1-NB $\left(^{\circ}\right) \quad$ Angle formed between the long axis of ma dibular incisor and the NB line

IMPA $\left(^{\circ}\right) \quad$ Angle formed by the extension of long axis of mandibular incisor to the mandibular plane

U1L1 $\left(^{\circ}\right) \quad$ Angle formed by the extensions of long axes of maxillary incisors to the mandibular incisors

$\mathrm{SN} / \mathrm{GoGn}\left(^{\circ}\right.$ Angle formed between the mandibular plane (GoGn) and the SN plane 
from pre-treatment, post-treatment, and post-retention phases. All cephalometric films were taken for each subject in centric occlusion with a relaxed and closed lip position using the same Sirona Orthophos XG (Bensheim, Germany) imaging system. Additionally, each subject's head was stabilized by positioning the ear rods of the machine in the external auditory meatus with the Frankfurt horizontal plane parallel to the horizontal and sagittal plane at right angles to the path of the X-ray (21). From the cephalometric analyses made using the NemoCeph NX 2005 (Nemotec, Madrid, Spain) program, dental and skeletal changes were evaluated. The angular and linear measurements used in this study are shown in Table 1.

In addition, the overjet, overbite, maxillary and mandibular intercanine widths and intermolar widths, arch lengths, and Little's irregularity indexes were measured on the dental models (Figure 2) $(22,23)$. The irregularity index defined by Dr. Little was calculated using the linear measurement of displacements in the anatomical contact points of maxillary and mandibular five anterior teeth, parallel to the occlusal plane (23). A digital caliper (Mitutoyo Corp., Kanagawa, Japan) with a $0.01 \mathrm{~mm}$ sensitivity was used for the measurements. All cephalometric analyses and dental model measurements were performed by the same investigator (MT).

\section{Statistical Analysis}

To assess the measurement precision, investigator reliability and intra-examiner agreement were calculated and found to be high (intraclass correlation coefficient $=0.890, \mathrm{p}<0.001$ ). In addition, the random measurement error was calculated with Dahlberg's formula, and it was observed that, for these linear and angular measurements, the error values ranged from 0.056 to 0.042 ( $\mathrm{mm}$ ) and $0.29^{\circ}$ to $0.14^{\circ}$, respectively.

In previous studies, it was observed that the standard deviation (s) ranged from 0.4 to 4 . For this reason, it was considered as 2

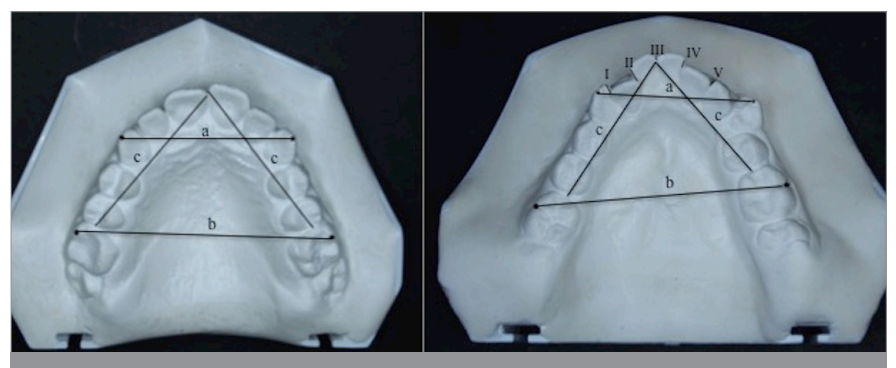

Figure 2. Dental model measurements. Irregularity index

$(I+I I+I I+I V+V)$; a, intercanine width; b, intermolar width; c, arch length in our study. In addition, the effect size (d) was assumed to be 1 , and the $Z$ value was 1.96 for a 0.05 Type I error rate. Then, the sample size was found to be 15.13 (@15) by using the equation of sample size calculation $\left(n=Z^{2} s^{2} / d^{2}\right)$.

Descriptive statistics for the continuous variables were presented as the mean, standard deviation, and minimum and maximum values, while counts and percentages were used for categorical variables. A repeated measures analysis of variance with two factors (time was the dependent factor, treatment was the independent factor) was used for comparing the groups and periods in terms of continuous variables. To identify the different groups, a Duncan multiple comparison test was calculated. The statistically significant level was considered to be $5 \%$, and the Statistical Package for Social Sciences (SPSS Inc.; Chicago, IL, USA) version 13.0 statistical program was used for all statistical computations.

\section{RESULTS}

In the Essix and Hawley groups, the mean age of patients was $17.53 \pm 3.89$ and $16.54 \pm 2.24$ years, respectively, and the mean treatment times were $2.90 \pm 0.62$ and $3.11 \pm 0.53$ years, respectively. No statistically significant difference was found among the groups in the number, mean age, and mean treatment times of patients (Table 2).

Pre-treatment, post-treatment, and post-retention maxillary and mandibular dental model measurements in the Essix and Hawley groups are shown in Table 3. There was no statistically significant difference between the groups and identified time periods in terms of the overjet, overbite, maxillary and mandibular intercanine widths, intermolar widths and arch lengths. In addition, although the maxillary and mandibular irregularity indexes increased from the post-treatment to the post-retention phase, the difference was not statistically significant.

Pre-treatment, post-treatment, and post-retention lateral cephalometric measurements for the Essix and Hawley groups are presented in Table 4. The lateral cephalometric measurements were not statistically significant between the groups and identified time periods.

\section{DISCUSSION}

Studies in the literature have recorded that there is no uniform retainer type recognized for a long-term stability, and the retainers and their wearing times showed variability $(24,25)$. It has

Table 2. Descriptive statistics

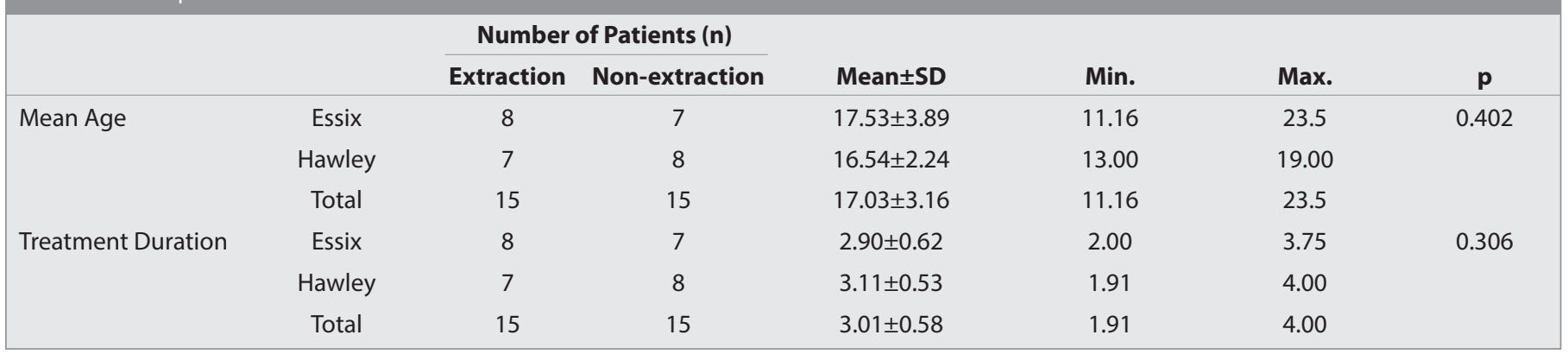


Table 3. Pre-treatment, post-treatment, and post-retention maxillary and mandibular dental model measurements

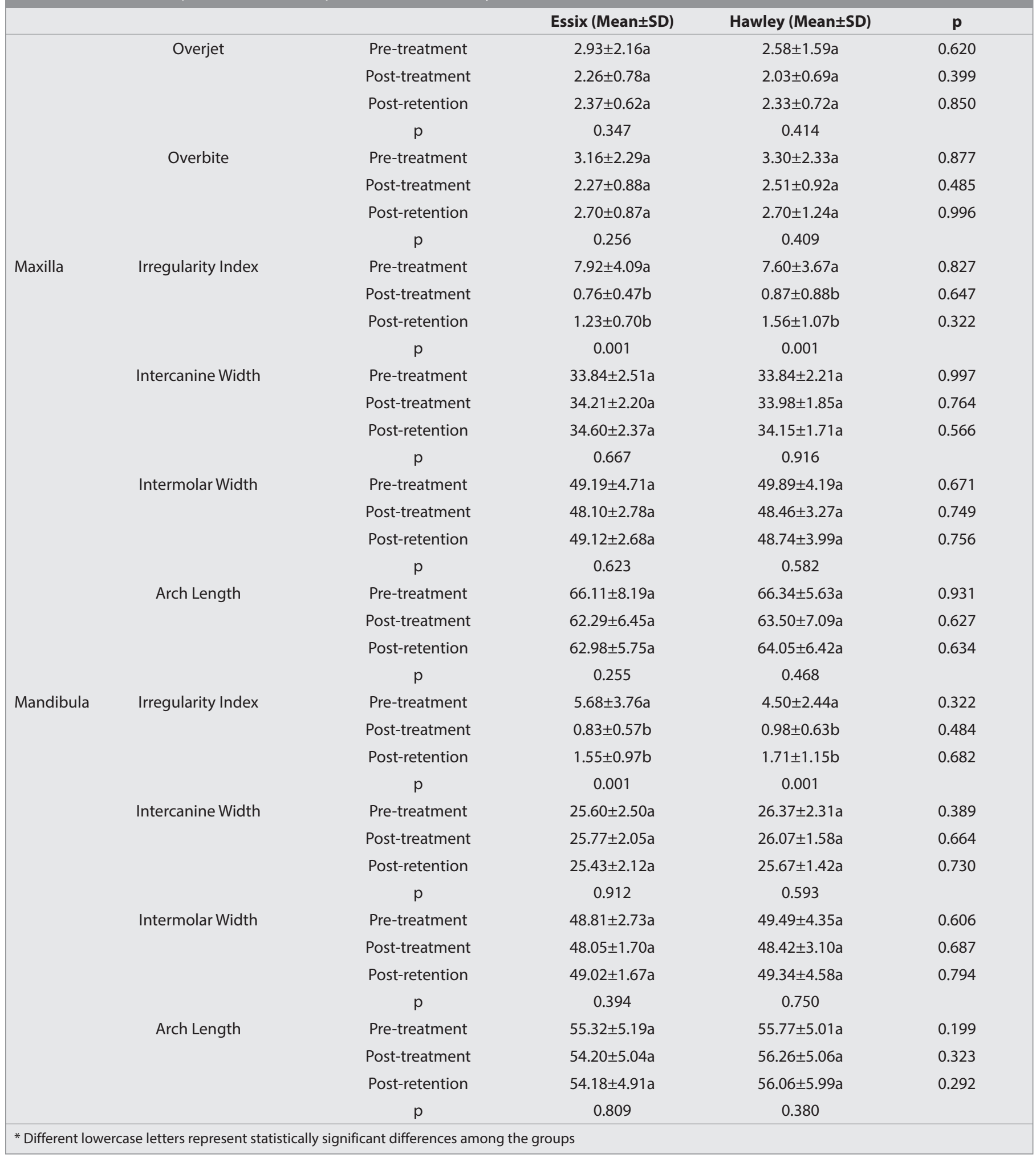

also been stated that relapse occurs independently from the retainer used; therefore, factors such as cost-effectiveness, patient comfort and satisfaction, settling, clinical effectiveness, ease of production, and survival time may be more important in the retainer selection $(5,8)$. The aim of this study was to compare the clinical effectiveness of the Essix and Hawley retainers that are frequently used in orthodontic practice $(5,16,17)$.
There are also conflicting opinions about the wearing times of the retainers. Among these, Ramazanzadeh et al. (11) concluded that, during the 8 months of a retention period for a better incisor alignment in the lower jaw, the retention protocols of 4 months full-time followed by night-only wear is better than 1 week full-time followed by night-only wear. However, Shawesh et al. (26) expressed that, in terms of the incisor irregularity index 
Table 4. Pre-treatment, post-treatment, and post-retention lateral cephalometric measurements

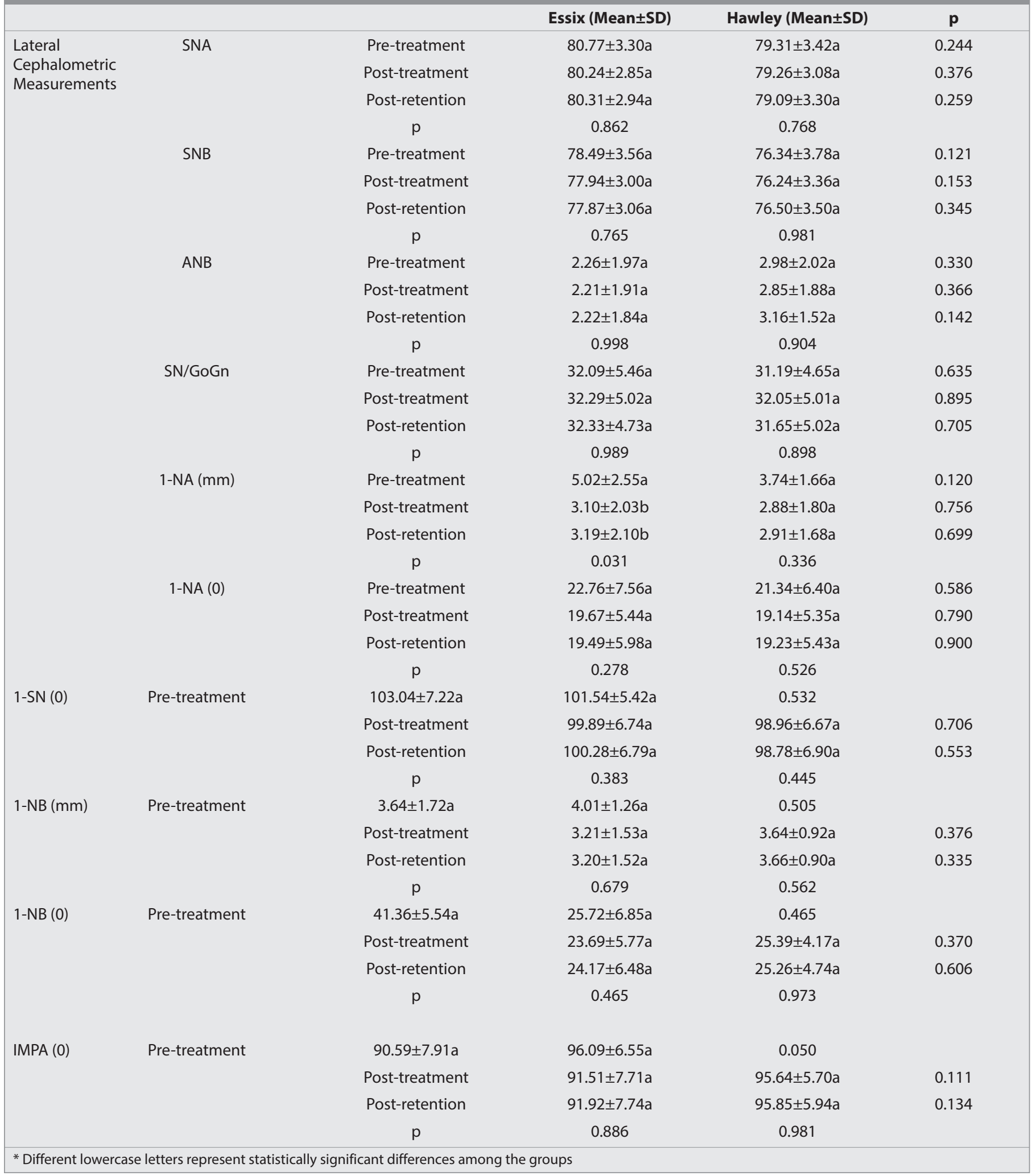

and incisor crowding, no significant difference was found between the retention protocols for night-time wear only for 1 year or 6 months full-time followed by 6 months of night-only wear. In addition, Proffit (4) stated that the retention period should be continued for at least 12 months, and by shortening the wearing time to 4-6 months after the post-treatment, it can be used only at night. With this information, in the present study, we also preferred a 1-year retention period with 6 months full-time and then 6 months at night only.

Based on the current studies, Meade and Millett (27) stated that orthodontists commonly recommend an Essix retainer sheet 
thicknesses of $0.75 \mathrm{~mm}$ and $1 \mathrm{~mm}$. In addition, Zhu et al. (28) found no significant difference between the Essix retainers of $0.75 \mathrm{~mm}$ and $1 \mathrm{~mm}$ thicknesses in terms of survival time, failure rate, and comfort. For this reason, the use of an Essix retainer sheet thickness of $1 \mathrm{~mm}$ (0.40 inch) was preferred in our study.

Although there is insufficient evidence to determine which retainer is more effective in studies comparing Essix and Hawley retainers $(5,6)$, the overjet, overbite, maxillary and mandibular intercanine widths, intermolar widths, arch lengths, and irregularity indexes were evaluated with regard to clinical effectiveness. Lindauer and Shoff (29) compared the overjet, overbite and Little's irregularity index over 6 months of the retention period. They found no statistically significant difference in evaluated parameters between the groups, although increased crowding was observed in the Hawley group for both dental arches. In two separate studies that evaluated the intercanine and intermolar widths, arch length, and irregularity indexes, Barlin et al. (30) found no statistically significant difference between the groups in the 2nd, 6th, and 12th month of the retention period. However, Ramazanzadeh et al. (11) concluded that the upper arch length and the upper-lower irregularity indexes were significantly lower in the Essix group during the 8th month of the retention period. In this study, we also found no significant difference in the overjet, overbite, intercanine and intermolar widths, and irregularity index between the Essix and Hawley groups during 1 year of the retention period.

Rowland et al. (16) compared the clinical effectiveness of Essix and Hawley retainers after the extraction or non-extraction fixed orthodontic treatment for 6 months. In the extraction group, Essix and Hawley retainers were applied to 68 and 66 of these subjects, respectively; in the non-extraction group, Essix and Hawley retainers were applied to 133 and 130 of these subjects, respectively. The authors observed no significant difference in the rotation and intercanine and intermolar widths between the groups, which was consistent with our results. However, in terms of Little's irregularity index, the Essix retainer was found to be more effective in both maxillary and mandibular labial segments than the Hawley retainer, especially in the lower arch. Similarly, Babacan et al. (31) compared the efficiency of Essix and Hawley retainers on mandibular anterior crowding in 40 non-extraction patients, using an irregularity index. At 1 year and 7 months after the treatment, a significant increase in irregularity indexes was found in both groups, but this increase was less in the Essix group. However, it was observed that there was no information about cephalometric measurements and arch lengths in these studies.

Demir et al. (17) compared the clinical effectiveness of Essix and Hawley retainers for a 1-year retention period and after a 2-year follow-up period in 42 patients who had non-extraction fixed orthodontic treatment. Consistent with our results, in all time periods, the authors found no statistically significant difference in intercanine widths and maxillary arch lengths in both groups and mandibular arch lengths in the Essix group. In the Hawley group, although the difference between the after treatment and 2-year follow-up period was statistically significant for mandibular arch length, the difference between other time periods was not significant. In terms of Little's irregularity index, the differences between all time periods in the Hawley group and between the after treatment and 1-year retention period in the Essix group were statistically significant. As a result, they concluded that the arch lengths that increased during orthodontic treatment tended to return to their pre-treatment value after the retention period, but this was significant only in the Hawley group. With Little's irregularity index, although the Essix retainer was more efficient in the mandibular anterior region during the retention period, the two retainers showed similar properties after a 2-year follow-up period. In addition, it was observed that there were slight changes in the cephalometric measurements between the two groups, and the upper-lower incisor inclinations, incisor positions, and arch lengths increased. We suggest that the upper-lower incisor inclinations, incisor positions, and arch lengths did not increase in our study in which extraction and non-extraction treatments were included and may be the reason for these different results.

Consistent with our results, Gómez-Gómez et al. (32) evaluated dental stability from the lateral cephalometric radiographs and found no statistically significant difference between the Essix and Hawley retainers during 6 months of the retention phase. However, in this study, they did not give any information about the pre-treatment cephalometric measurements.

The arch lengths gradually decreased due to the physiological migration of teeth, and anterior crowding may have occurred even in the case of third molar deficiency, especially in the lower arch (33). Additionally, the preservation of pre-treatment arch forms is very important to obtain the best long-term stability since the increased intercanine and intermolar widths during treatment tended to decrease after the retention period (34). For this reason, it has been stated that even if a good and well-functioning occlusion is obtained with orthodontic treatment, relapse may be seen after years of treatment, and patients should be informed of this.

A small sample size, no post-retention follow-up periods, and the investigation of only two retention protocols were the main limitations of this study. For this reason, conducting new studies with larger sample sizes, longer follow-up periods, and different retainer types used after extraction and non-extraction treatment is recommended.

\section{CONCLUSION}

- The differences between the Essix and Hawley retainers in the overjet, overbite, maxillary and mandibular intercanine widths, intermolar widths, and arch lengths were not statistically significant.

- $\quad$ Although the maxillary and mandibular irregularity indexes increased from the post-treatment to the post-retention phase, the difference was not statistically significant.

- In terms of pre-treatment, post-treatment, and post-retention lateral cephalometric measurements, no statistically significant difference was found between and within the groups. 
Ethics Committee Approval EEthics committee approval was received for this study from the Ethics Committee of Van Yüzüncü Yıl University School of Medicine (B.30.2.YYU.0.01.00.00/125).

Informed Consent: Written informed consent was obtained from the patients who participated in this study.

Peer-review: Externally peer-reviewed.

Author Contributions: Concept - Y.K.; Design - Y.K.; Data Collection and/ or Processing - Y.K., M.T.; Analysis and/or Interpretation - Y.K., S.K.; Literature Search - Y.K.; Writing Manuscript - Y.K.; Critical Review - Y.K.

Conflict of Interest: The authors have no conflict of interest to declare.

Financial Disclosure: The authors declared that this study has received no financial support.

\section{REFERENCES}

1. Valladares-Neto J, Evangelista K, Miranda de Torres H, Melo Pithon M, Alves Garcia Santos Silva M. A 22-year follow-up of the nonsurgical expansion of maxillary and mandibular arches in a young adult: Are the outcomes stable, relapsed, or unstable with aging? Am J Orthod Dentofacial Orthop 2016; 150: 521-32. [CrossRef]

2. Melrose C, Millett DT. Toward a perspective on orthodontic retention? Am J Orthod Dentofacial Orthop 1998; 113: 507-14. [CrossRef]

3. Al Rahma WJ, Kaklamanos EG, Athanasiou AE. Performance of Hawley-type retainers: a systematic review of randomized clinical trials. Eur J Orthod 2018; 40: 115-25. [CrossRef]

4. Proffit WR. Retention. Proffit WR, Fields H, Sarver D, editors. Contemporary Orthodontics. Mosby: Elsevier; 2012.p.606-16.

5. Mai W, He J, Meng H, Jiang Y, Huang C, Li M, et al. Comparison of vacuum-formed and Hawley retainers: a systematic review. Am J Orthod Dentofacial Orthop 2014; 145: 720-7. [CrossRef]

6. Littlewood SJ, Millett DT, Doubleday B, Bearn DR, Worthington HV. Retention procedures for stabilising tooth position after treatment with orthodontic braces. Cochrane Database Syst Rev 2016; 29: CD002283. [CrossRef]

7. Al-Moghrabi D, Pandis N, Fleming PS. The effects of fixed and removal orthodontic retainers: a systematic review. Prog Orthod 2016; 17: 24. [CrossRef]

8. Luther F, Nelson-Moon Z, editors. Orthodontic retainers and removable appliances: Principles of design and use. Oxford-UK: Wiley-Blackwell; 2013.

9. Swidi AJ, Taylor RW, Tadlock LP, Buschang PH. Recent advances in orthodontic retention methods: A review article. J World Fed Orthod 2018; 7: 6-12. [CrossRef]

10. Zachrisson BU. Clinical experience with direct bonded orthodontic retainers. Am J Orthod 1977; 71: 440-8. [CrossRef]

11. Ramazanzadeh B, Ahrari F, Hosseini ZS. The retention characteristics of Hawley and vacuum-formed retainers with different retention protocols. J Clin Exp Dent 2018; 10: 224-31. [CrossRef]

12. Manzon L, Fratto G, Rossi E, Buccheri A. Periodontal health and compliance: A comparison between Essix and Hawley retainers. Am J Orthod Dentofacial Orthop 2018; 153: 852-60. [CrossRef]

13. Hichens $L$, Rowland $H$, Williams A, Hollinghurst $S$, Ewings $P$, Clark $S$, et al. Cost-effectiveness and patient satisfaction: Hawley and vacuum-formed retainers. Eur J Orthod 2007; 29: 372-8. [CrossRef]

14. Hoybjerg AJ, Currier GF, Kadioğlu O. Evaluation of 3 retention protocols using the American Board of Orthodontics cast and radiograph evaluation. Am J Orthod Dentofacial Orthop 2013; 144: 1622. [CrossRef]
15. Moslemzadeh SH, Sohrabi A, Rafighi A, Ghojazadeh M, Rahmanian S. Comparison of survival time of Hawley and Vacuum-formed retainers in orthodontic patients - a randomized clinical trial. ABCMed 2017; 5: 7-15. [CrossRef]

16. Rowland $H$, Hichens $L$, Williams A, Hills D, Killingback N, Ewings $P$, et al. The effectiveness of Hawley and vacuum-formed retainers: a single-center randomized controlled trial. Am J Orthod Dentofacial Orthop 2007; 132: 730-7. [CrossRef]

17. Demir A, Babacan H, Nalcacı R, Topcuoglu T. Comparison of retention characteristics of Essix and Hawley retainers. Korean $\mathrm{J}$ Orthod 2012; 42: 255-62. [CrossRef]

18. Xu XC, Li RM, Tang GH. Clinical evaluation of lingual fixed retainer combined with hawley retainer and vacuum-formed retainer. Shanghai Kou Qiang Yi Xue 2011; 20: 623-6.

19. Moslemzadeh SH, Sohrabi A, Rafighi A, Farshidnia S. Comparison of stability of the results of orthodontic treatment and gingival health between Hawley and vacuum-formed retainers. J Contemp Dent Pract 2018; 19: 443-9. [CrossRef]

20. Sheridan JJ, LeDoux W, McMinn R. Essix retainers: fabrication and supervision for permanent retention. J Clin Orthod 1993; 27: 3745.

21. Basciftci FA, Uysal T, Buyukerkmen A. Craniofacial structure of Anatolian Turkish adults with normal occlusions and well-balanced faces. Am J Orthod Dentofacial Orthop 2004; 125: 366-72. [CrossRef]

22. Huth J, Staley RN, Jacobs R, Bigelow $H$, Jakobsen J. Arch widths in class II-2 adults compared to adults class I- 1 and normal occlusion. Angle Orthod 2007; 77: 837-44. [CrossRef]

23. Little RM. The irregularity index: a Quantitative score of mandibular anterior alignment. Am J Orthod 1975; 68: 554-63. [CrossRef]

24. Dindaroglu F. Is retention necessary? Türkiye Klinikleri J Orthod-Special Topics 2017; 3: 73-8.

25. Padmos JAD, Fudalej PS, Renkema AM. Epidemiologic study of orthodontic retention procedures. Am J Orthod Dentofacial Orthop 2018; 153: 496-504. [CrossRef]

26. Shawesh M, Bhatti B, Usmani T, Mandall N. Hawley retainers full- or part-time? A randomized clinical trial. Eur J Orthod 2010; 32: 16570. [CrossRef]

27. Meade MJ, Millett D. Retention protocols and use of vacuum-formed retainers among specialist orthodontists. J Orthod 2013; 40:318-25. [CrossRef]

28. Zhu Y, Lin J, Long $H$, Ye N, Huang R, Yang $X$, et al. Comparison of survival time and comfort between 2 clear overlay retainers with different thicknesses: a pilot randomized controlled trial. Am J Orthod Dentofacial Orthop 2017; 151: 433-9. [CrossRef]

29. Lindauer SJ, Shoff RC. Comparison of Essix and Hawley retainers. J Clin Orthod 1998; 32: 95-7.

30. Barlin S, Smith R, Reed R, Sandy J, Ireland AJ. A retrospective randomized double-blind comparison study of the effectiveness of Hawley vs vacuum-formed retainers. Angle Orthod 2011; 81: 404-9. [CrossRef]

31. Babacan H, Bıcakcı AA, Doruk C, Demir A. Essix ve Hawley pekiştirme apareylerinin mandibular anterior çapraşıklık nüksüne etkileri. Cumhuriyet Dent J 2002; 5: 63-5.

32. Gómez-Gómez SL, Salazar-Quiceno LF, Guisao DA, Betancur-Pérez $\mathrm{JJ}$, Segura-Cardona AM. Effectiveness of two retainer types in dental and occlusal stability. Rev Fac Odontol Univ Antioq 2016; 28: 3453. [CrossRef]

33. Thilander B. Dentoalveoler development in subjects with normal occlusion. A longitudional study between ages of 5 and 31 years. Eur J Orthod 2009; 31: 109-20. [CrossRef]

34. Steinnes J, Johnsen G, Kerosuo H. Stability of orthodontic treatment outcome in relation to retention status: An 8-year follow-up. Am J Orthod Dentofacial Orthop 2017; 151: 1027-33. [CrossRef] 